\title{
The utility of molecular genetic techniques in craniosynostosis cases associated with intellectual disability
}

Alina Bogliş ${ }^{1,2,3^{*}}$, Florin Tripon ${ }^{2,3}$, Claudia Bănescu ${ }^{1,2,3}$

1. Laboratory of Genetics, Emergency Clinical County Hospital Târgu Mureș, Romania

2. Department of Genetics, University of Medicine, Pharmacy, Sciences and Technology of Târgu Mureş, Romania

3. Laboratory of Molecular Biology/Genetics, CCAMF, University of Medicine, Pharmacy, Sciences and Technology of Târgu Mureş, Romania

\begin{abstract}
Molecular genetic testing in craniosynostosis leads to the detection of the mutations in the genes encoding fibroblast growth factor receptors (FGFR), providing information about the etiology of the genetic disorder. Muenke syndrome is produced by p.Pro250Arg mutation in FGFR3 gene with evidence of variable expressivity, representing $8 \%$ of the syndromic craniosynostoses.

Here, we present the identification of a p.Pro250Arg pathogenic mutation (c.749C>G) in the FGFR3 gene using Multiplex Ligation-dependent Probes Amplification (MLPA) analysis in conjunction with Sanger sequencing in a patient with craniosynostosis and mild intellectual disability. The MLPA analysis detected a reduced signal of the probe, at the site of the c.749C $>G$ mutation, defined by the presence of one allele of $C 749>G$ mutation in the FGFR3 gene, exon 7. Sanger sequencing was performed for confirmation and identified heterozygous p.Pro250Arg pathogenic variant (c.749C>G) in exon 7 of the FGFR3.

In conclusion, we assessed the validity and clinical utility of the combined molecular genetic techniques, MLPA analysis, and Sanger sequencing, for craniosynostosis and intellectual disability, improving not only the diagnostic testing but also the genetic counseling and management of the disorder.
\end{abstract}

Keywords: craniosynostosis, FGFR3, Pro250Arg mutation, MLPA analysis, Sanger sequencing

Received: $29^{\text {th }}$ July 2018; Accepted: $18^{\text {th }}$ September 2018; Published: $8^{\text {th }}$ October 2018

\section{Introduction}

In the last decades, molecular genetic techniques have been developed to confirm the genetic etiology of many genetic disorders $(1,2)$. Nowadays, the genetic etiology of the most frequent genetic disorders has been reported, and a causative gene mutation is identified in approximately $24 \%$ of patients with craniosynostosis (1). The most common craniosynostosis syndromes, are the Crouzon syndrome, Saethre-Chotzen syndrome, Apert syndrome, Pfeiffer syndrome, and Muenke syndrome, but sometimes these syndromes are difficult to diagnose clinically, especially those with a clinically vari-

* Corresponding author: Alina Bogliş, University of Medicine, Pharmacy, Sciences and Technology of Târgu Mureş, Romania. E-mail: alinaboglis@gmail.com; alina.boglis@umftgm.ro 
able phenotype; therefore all cases require molecular genetic testing (3).

Muenke syndrome (MS) (OMIM \# 602849), an autosomal dominant disorder, produced by p.Pro250Arg mutation in the fibroblast growth factor receptor 3 (FGFR3) gene is the most common craniosynostosis syndrome $(4,5)$. The incidence of MS is about 1 in 30,000 individuals and represents $8 \%$ of the syndromic craniosynostoses $(6,7)$. It shows that MS has an incomplete penetrance of the mutation of approximately $80 \%(8,9)$.

MS is characterized by unicoronal or bicoronal craniosynostosis (7), with a highly variable phenotype from normal appearance or isolated craniosynostosis, macrocephaly, to more complex features that can resemble with another craniosynostosis, such as Crouzon syndrome, Saethre-Chotzen or Pfeiffer syndrome.

Despite the huge progress in both clinical and molecular genetic aspects, choosing the right genetic test can be challenging, because of financial limitations in some regions or availability of these techniques. In the last two decades, Multiplex Ligation-dependent Probes Amplification (MLPA) became more available, which seems to be able to identify an increasing number of cases compared with Fluorescence in Situ Hybridization (FISH) and Comparative Genomic Hybridization (CGH) (10). However, these standard approaches have an essential role in specific genetic disorders. Furthermore, gene sequencing may represent a second-tier genetic testing when MLPA is available because sequencing is less cost-effective, taking into account the genetic variability of the mutations and overlapping features, molecular testing being limited to a single gene or a few at the same time (1).

Here, we present the identification of a p.Pro250Arg mutation (c.749C $>$ G) in the FGFR3 gene using MLPA analysis in conjunction with Sanger sequencing in a patient with craniosynostosis and mild intellectual disability.

\section{Material and methods}

\section{Subject}

An 11-year-old male patient with Crouzon syndrome suspicion was referred to the Genetics Laboratory of the Clinical Emergency County Hospital, Tîrgu Mureș, Romania. The patient was diagnosed at the age of 5 weeks with craniosynostosis and later on with mild intellectual disability. The craniosynostosis was confirmed by physical examination, skull radiography, and computed tomography scan. Family history is negative for the similar complaints, with no suspicion of consanguinity between the healthy parents; his older sister has Turner syndrome with karyotype 45,X. Before blood sampling, the patient's mother gave us informed written consent for the molecular genetic testing. Ethical approval for the genetic testing was granted by the Ethics Committee from the University of Medicine and Pharmacy Tîrgu Mureș, Romania.

\section{Molecular genetic techniques}

Fresh peripheral blood $(2 \mathrm{ml})$ was collected into EDTA vacutainer tube from the patient and DNA was isolated and purified using the Quick-gDNA MiniPrep kit (Zymo Research, USA) following the manufacturer's instructions, with a DNA concentration of $102.68 \mathrm{ng} / \mu 1$, and stored at $4^{\circ} \mathrm{C}$ for further analysis.

\section{MLPA analysis}

First, the genomic DNA sample was evaluated through MLPA analysis by using SALSA MLPA probemix P080-C1 CRANIOFACIAL (MRC-Holland $\AA$, Amsterdam, Netherlands). The kit contains probes for the FGFR1, FGFR2, FGFR3, MSX2, TWIST1, ALX1, ALX3, ALX4, EFNB1 and RUNX2 genes (www.mlpa.com) to detect deletion or duplication of one or several exons and certain point mutations. The results were interpreted with the Coffalyser software, 
available from the MRC-Holland ${ }^{\circledR}$. The MLPA analysis detected a reduced signal of one of the analyzed probes which suggested a heterozygous mutation in exon 7 of the FGFR3 gene.

\section{Sanger sequencing and interpretation analysis}

For confirmation of the results obtained by MLPA analysis, Sanger sequencing was carried out using BigDye Terminator v3.1 Cycle Sequencing Kit (Applied Biosystems, Foster City, CA, USA) and analyzed by capillary electrophoresis on the Applied Biosystems ${ }^{\mathrm{TM}} 3500$ Dx Series Genetic Analyzer.

Amplification of genomic DNA sample was performed using previously described primers for exon 7 in FGFR3 gene, forward: GACGTACACGCTGGACGTGCT, and reverse: ACGCAGCTGCCTATGGCCCTGA(9). Polymerase Chain Reaction (PCR) conditions set up in our laboratory were: an initial 10 minutes at $95^{\circ} \mathrm{C}$, succeeded by 35 cycles at $95^{\circ} \mathrm{C}$ for 45 seconds, 45 seconds for annealing at $58^{\circ} \mathrm{C}, 1$ minute at $72^{\circ} \mathrm{C}$, after that for final extension at $72^{\circ} \mathrm{C}$ for about 7 minutes.

PCR products were purified using the CleanSweep ${ }^{\mathrm{TM}}$ PCR Purification reagent. Direct DNA sequencing of both strands was performed using BigDye ${ }^{\mathrm{TM}}$ Terminator v3.1 Cycle Sequencing Kit. To purify sequencing reactions, we used BigDye XTerminator ${ }^{\mathrm{TM}}$, and after that, the sequencing reactions were loaded into the genetic analyzer. The sequences were analyzed using Variant Reporter ${ }^{\mathrm{TM}}$ Software v1.1 (Applied Biosystems).

The molecular genetics techniques were conducted at the Laboratory of Molecular Biology/Genetics at the Advanced Research Center Medical and Pharmaceutical from the University of Medicine and Pharmacy of Tîrgu Mureș.

\section{Results}

\section{Clinical findings}

The clinical features of our patient resemble Crouzon syndrome: turribrachycephaly, exophthalmos with left microphthalmia, hypertelorism, hypoplastic maxilla, beaked shape nose, higharched palate, ectopic eruption teeth 13, abnormal dental occlusion, low-set ears, short upper lip, proximal syndactyly of toes two and three. Regarding other physical examinations, the patient was found to be normal. Also, our patient presented a mild intellectual disability.

\section{Molecular genetic techniques}

The MLPA analysis detected a reduced signal of the probe, at the site of the c.749C $>\mathrm{G}$ (p.Pro250Arg) mutation, defined as the presence of one allele of $\mathrm{C} 749>\mathrm{G}$ mutation in the FGFR3 gene, exon 7. MLPA analysis was performed twice with similar results, the value of the reduced signal (dosage quotient of the reference probes in our patient DNA sample) was $<0.65$ (normal value is between 0.80 and 1.20). These findings should always be validated by other molecular techniques, according to the MRC-Holland $\AA$ recommendation (Figure 1).

Sanger sequencing identified heterozygous p.Pro250Arg mutation (c.749C $>$ G) in our patient. This missense variant, a single nucleotide substitution (c.749C $>$ G) leads to a substitution of arginine to proline at amino acid 250 of the FGFR3 gene (Figure 2).

\section{Discussions}

Given that craniosynostoses are a heterogeneous group of disorders that may include overlapping features, making the correct clinical diagnosis can be challenging. Over the years, several studies tried to identify the genes and the mechanisms for craniosynostosis and developed a more efficient and cost-effective molecular diagnostic strategy for genetic testing, where 


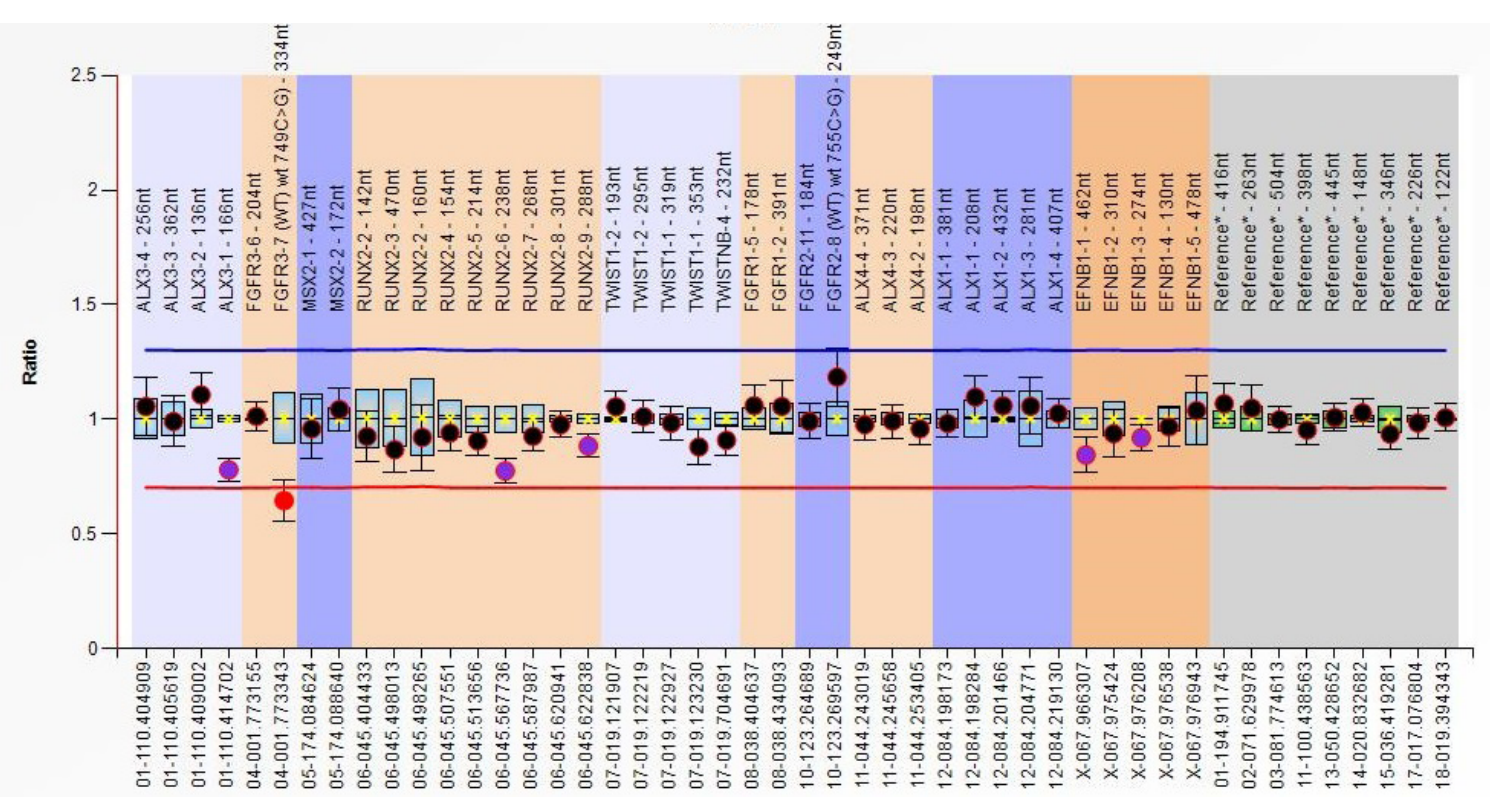

Fig. 1. MLPA analysis: red color indicates the reduced signal of c.749C $>$ G, the FGFR3 gene $($ ratio $<0.65)$

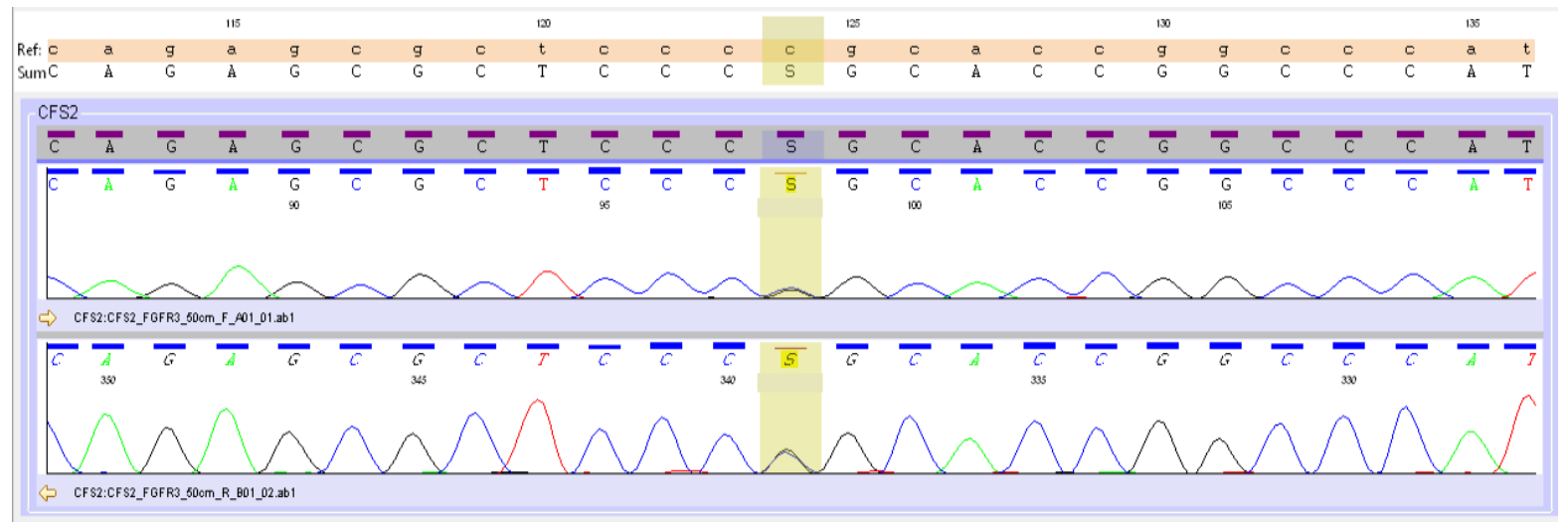

Fig. 2. Electropherogram of Sanger sequencing: marked green section indicates a heterozygous c.749C $>$ G (C to G) pathogenic mutation in the FGFR3 gene, where $\mathrm{S}$ symbol is a single-letter code recommendation of nucleotides, meaning $\mathrm{G}$ or $\mathrm{C}$

gene-targeted sequencing of recurrent mutations was carried out $(1,8,11,12)$. Many studies demonstrated in both humans and mice the implication of the FGFR1, FGFR2, FGFR3, FBN1, MSX2, and TWIST genes in the morphogenesis of the cartilage and bones $(13,14)$.

A heterozygous p.Pro250Arg mutation of the FGFR3 gene has been found in our patient with craniosynostosis and mild intellectual disability. The patient with a Crouzon syndrome resemble phenotype was at first investigated by MLPA analysis, where a reduced signal probe has been identified in the position of c. $749 \mathrm{C}>\mathrm{G}$ of the FGFR3 gene. This mutation is particular to MS and sometimes the phenotype of MS is overlapped with other syndromic craniosynos- 
toses $(9,15,16)$. Furthermore, in the phenotypic spectrum for MS, besides craniosynostosis, are included: midfacial hypoplasia, hypertelorism, brachydactyly, coned epiphyses, carpal and tarsal fusion, low-set ears, hearing loss, epilepsy, developmental delay, behavior problems, and mild intellectual disability $(9,15,17)$.

To validate the p.Pro250Arg mutation of the FGFR3 gene, identified through MLPA analysis, we carried out the Sanger sequencing of the FGFR3 gene in our patient. Gene-targeted sequencing for the c.749C $>\mathrm{G}$ in exon 7 of the FGFR3 gene has confirmed the presence of the heterozygous p.Pro250Arg mutation, defined as a single nucleotide substitution. According to the last evaluation of the NCBI ClinVar (www.ncbi.nlm.nih.gov/clinvar/), the references NM_00142.2(FGFR3):c.749C $>$ G (p.Pro250Arg) with the cytogenetic location 4 p16 and genomic location at the level Chr4: 1801844, on Assembly GRCh38, is considered to be a pathogenic variant associated with MS.

Because of the phenotype similarities of MS with other craniosynostosis syndromes caused by different mutations in the FGFR3 gene, the direct gene-targeted sequencing is rarely performed (11). There are studies where the exome and whole genome sequencing (WGS) were used to identify the Pro250Arg mutation of the FGFR3 gene as the initial genetic testing $(1,8)$, but this implies considerable financial resources. Even though mutations in the FGFR1, FGFR2, FGFR3, FBN1, MSX2, and TWIST genes represent $3 / 4$ from all diagnosed cases, the etiology of the remaining craniosynostosis varies. Thus, whole exome sequencing (WES), as compared to Sanger sequencing, is useful to determine mutations in FGFR genes in heterogeneous disorders $(13,18)$.

Therefore, according to the recommendation of Hehr U (19), genetic testing should start with an investigation of exon 7 for the detection of the Pro250Arg mutation of the FGFR3 gene, and in the case of other mutations suggested by the patients' phenotype, MLPA followed by sequencing analysis is recommended.

For example, successful detection of a single point mutation in the FGFR3 gene is the p.Pro250Arg mutation in our patient using MLPA analysis, as routine molecular testing to establish an accurate diagnosis. The results of the MLPA analysis performed helped us to select the exon for DNA sequencing saving time and financial resources. Confirmation through Sanger sequencing of the associated mutation is essential for proper clinical management for optimal care (for example, neurodevelopment evaluation, screening for neurosensorial hearing loss, ophthalmologic evaluation) and genetic counseling of the family with estimation of recurrence risk for future pregnancies and testing other family members at risk (20).

MLPA, a multiplex technique, allows identification of deletions and duplications of the several DNA sequences in the same reaction (www. mlpa.com) or chromosomal changes of interest that remain undetected using other molecular techniques, like DNA sequencing $(21,22)$. Also, the MLPA method may be considered as a good alternative to array-based techniques for many genetic disorders for the routine application (www.mlpa.com). MLPA analysis is a rapid method and a very reliable and cost-efficient test for the genetic diagnosis of craniosynostosis or other genetic disorders associated with craniosynostosis, intellectual disability of unknown etiology, but always a correct clinical diagnosis is mandatory to enhance the genetic diagnosis efficiency.

The use of MLPA panel allowed us to establish the correct diagnosis identifying the c. 749 $\mathrm{C}>\mathrm{G}$ mutation in a patient with craniosynostosis and intellectual disability, and we validated and extended the MLPA findings performing Sanger sequencing. Sequencing analyses are typically not recommended as an initial diag- 
nostic method as far as mutations are not easily found using this molecular test. Instead, Sanger sequencing is generally used to confirm a mutation found by another method.

Although the Sanger sequencing is not available at a large clinical scale for diagnosis because of the high costs, the MLPA analysis remains a reliable and cost-effective primary test for detection of the DNA sequence changes to establish the causes of numerous syndromes or intellectual disability.

In conclusion, we assessed the validity and clinical utility of the combined molecular genetic techniques, MLPA analysis and Sanger sequencing for craniosynostosis associated with intellectual disability, improving not only the diagnostic testing but also the genetic counseling and management of the disorder.

\section{Conflict of interest}

Conflicts of interest: none

\section{Authors' contributions}

$\mathrm{AB}$ : drafting the original manuscript, performed sequencing analysis and interpretation of the results

FT: performed sequencing analysis and interpretation of the results, review the final version of the manuscript

CB: performed MLPA analysis, critical revision, final approval of the draft to be published
Abbreviations
MS - Muenke syndrome
FGFR3 - fibroblast growth factor receptor 3
CGH - Comparative Genomic Hybridization
MLPA - Multiplex Ligation-dependent Probes
Amplification
EDTA - Ethylenediaminetetraacetic acid
DNA - Deoxyribonucleic acid
PCR - Polymerase Chain Reaction

NCBI - National Center for Biotechnology Information

\section{References}

1. Miller K, Twigg S, McGowan S, Phipps J, Fenwick A, Johnson D, et al. Diagnostic value of exome and whole genome sequencing in craniosynostosis. J Med Genet. 2017 Apr;54(4):260-8. DOI: 10.1136/jmedgenet-2016-104215

2. Negura L, Negura A. Sanger sequencing of MMR genes in a one-plate system. Rev Romana Med Lab. 2018 Apr;26(2):153-63. DOI: 10.2478/rrlm-2018-0008

3. Addissie, Yarnell C, Kruszk P, Muenke M. Muenke syndrome. Middle East J Med Genet. 2015 Jan;4:1-6. DOI: 10.1097/01.MXE.0000456629.07295.8e

4. Bellus G, Gaudenz K, Zackai E, Clarke L, Szabo J, Francomano $\mathrm{C}$, et al. Identical mutations in three different fibroblast growth factor receptor genes in autosomal dominant craniosynostosis syndromes. Nat Genet. 1996 Oct;14(2):174-6. DOI: 10.1038/ng1096-174

5. Muenke M, Gripp K, McDonald-McGinn D, Gaudenz $\mathrm{K}$, Whitaker LA L, Bartlett S, et al. A unique point mutation in the fibroblast growth factor receptor 3 gene (FGFR3) defines a new craniosynostosis syndrome. Am J Hum Genet. 1997 Mar;60(3):555-64.

6. Boulet S, Rasmussen S, Honein M. A population-based study of craniosynostosis in metropolitan Atlanta, 19892003. AmJ Med Genet. 2008 Apr;146A(8):984-91. DOI: 10.1002/ajmg.a.32208

7. Kruszka P, Addissie Y, Yarnell C, Hadley D, Guillen Sacoto M, Platte P, et al. Muenke syndrome: An international multicenter natural history study. Am J Med Genet A. 2016 Apr;170A(4):918-29. DOI: 10.1002/ ajmg.a.37528

8. Heuzé Y, Holmes G, Peter I, Richtsmeier J, Jabs E. Closing the Gap: Genetic and Genomic Continuum from Syndromic to Nonsyndromic Craniosynostoses. Curr Genet Med Rep. 2014 Sep;2(3):135-45. DOI: 10.1007/s40142-014-0042-X

9. Yu J, Park D, Yoon S. A Korean Family with the Muenke Syndrome. J Korean Med Sci. 2010 Jul;25(7):10869. DOI: $10.3346 / \mathrm{jkms} .2010 .25 .7 .1086$

10. Yoon A, Pham B, Dipple K. Genetic Screening in Patients with Craniofacial Malformations. J Pediatr Genet. 2016 Dec;5(4):220-4. DOI: 10.1055/s-0036-1592423

11. Wilkie A, Johnson D, Wall S. Clinical Genetics of Craniosynostosis. Curr Opin Pediatr. 2017 Dec;29(6):6228. DOI: 10.1097/MOP.0000000000000542

12. Lee E, Le T, Zhu Y, Elakis G, Turner A, Venselaar H, et al. A craniosynostosis massively parallel sequencing panel study in 309 Australian and New Zealand patients: findings and recommendations. Genet Med. 2017. DOI: $10.1038 / \operatorname{gim} .2017 .214$ 
13. Twigg S, Wilkie A. A Genetic-Pathophysiological Framework for Craniosynostosis. Am J Hum Genet. 2015 Sep;97(3):359-77. DOI: 10.1016/j. ajhg.2015.07.006

14. Nah H, Koyama E, Agochukwu N, Bart S, Muenke M. Phenotype profile of a genetic mouse model for Muenke syndrome. Childs Nerv Syst. 2012 Sep;28(9):148393. DOI: $10.1007 / \mathrm{s} 00381-012-1778-9$

15. Doherty E, Lacbawan F, Hadley D, Brewer C, Zalewski C, Kim H, et al. Muenke syndrome (FGFR3-related craniosynostosis): expansion of the phenotype and review of the literature. Am J Med Genet A. 2007 Dec;143A(24):3204-15. DOI: 10.1002/ajmg.a.32078

16. Escobar L, Hiett A, Marnocha A. Significant phenotypic variability of Muenke syndrome in identical twins. Am J Med Genet A. 2009 Jun;149A(6):1273-6. DOI: 10.1002/ajmg.a.32841

17. Solomon B, Muenke M. Muenke Syndrome. In Muenke M, Kress W, Collmann H, Solomon B, editors. Craniosynostoses: Molecular Genetics, Principles of Diagnosis, and Treatment. Monogr Hum Genet. Basel: Karger; 2011:89-97. DOI: 10.1159/000318407

18. Hamilton A, Tétreault M, Dyment D, Zou R, Kernohan
$\mathrm{K}$, Geraghty M, et al. Concordance between whole-exome sequencing and clinical Sanger sequencing: implications for patient care. Mol Genet Genomic Med. 2016 May;4(5):504-12. DOI: 10.1002/mgg3.223

19. Hehr U. Molecular Genetic Testing of Patients with Craniosynostosis. In Muenke M, Kress W, Collmann H, Solomon B, editors. Craniosynostoses: Molecular Genetics, Principles of Diagnosis, and Treatment. Monogr Hum Genet. Basel: Karger; 2011:177-83. DOI: 10.1159/000318428

20. Phipps J, Skirton H. A Qualitative Study to Explore the Views and Attitudes towards Prenatal Testing in Adults Who Have Muenke Syndrome and their Partners. J Genet Couns. 2017 Oct;26(5):1130-42. DOI: 10.1007/ s10897-017-0094-7

21. Katsanis S, Katsanis N. Molecular genetic testing and the future of clinical genomics. Nat Rev Genet. 2013 Jun;14(6):415-26. DOI: 10.1038/nrg3493

22. Sireteanu A, Popescu R, Braha E, Bujoran C, Butnariu L, Caba L, et al. Detection of chromosomal imbalances using combined MLPA kits in patients with syndromic intellectual disability. Rev Romana Med Lab. 2014 Jun;22(2):157-64. DOI: 10.2478/rrlm-2014-0019 